\title{
Low-frequency oscillations while magnetic storms as a tool to determine the types of solar plasma flows
}

\author{
N. A. Barkhatov, S.E. Revunov, O. T. Cherney, Zh. V. Smirnova, and M. V. Mukhina
}

Minin Nizhny Novgorod State Pedagogical University (Minin University), Nizhny Novgorod, Russian

\begin{abstract}
Comparison of wavelet spectrum (skeletons) local maxima for disturbed components of solar plasma flow parameters and geomagnetic field disturbances recorded along the meridional station chain during geomagnetic storm intervals is performed in the range of magnetohydrodynamic (MHD) waves. An algorithm for quantitative evaluation of analyzed skeletons consistency has been developed. It has been used to demonstrate the possibility of the type of solar wind plasma flow elaboration on unique spectral signs of Solar wind speed disturbances, density and interplanetary magnetic field. It is shown that the energy spectrum of oscillations for these parameters reflects the internal structure of the corresponding plasma formation. The skeletons application to the analysis of the interplanetary space main parameters made it possible to estimate the magnetosphere reaction time in geomagnetic field horizontal component oscillations at different latitudes on the disturbance. As a result, the distributed magnetosphere reaction over latitude was determined in the form of geomagnetic field oscillations on the disturbed solar flow parameters. It is shown that the dynamics of the components of the solar wind parameters disturbance spectra corresponding to plasma flows manifest themselves in the MHD spectra of high-latitude stations magnetograms and can be used as a diagnostic tool.
\end{abstract}

\section{Introduction}

A parallel analysis of postprocessing results for wavelet components of low-frequency magnetohydrodynamic (MHD) disturbed solar wind plasma flows parameters and geomagnetic field horizontal component disturbances (geomagnetic pulsation range) recorded along the meridional stations chain during different geomagnetic storms was performed. Geomagnetic pulsations are short-period MHD oscillations in the range of $0.001-1 \mathrm{~Hz}$, excited in the Earth's magnetosphere and in the Solar wind. At the same time it is known that low-frequency (LF) oscillations part of the solar wind parameters penetrates into magnetosphere and appears there in magnetograms recordings of ground-based observatories [1]. This is possible when conditions for the transition region lightening up beyond the earth's shock wave are created for them [2]. This study verifies the assumption that during geomagnetic storms periods caused by solar plasma flows, some magnetic LF disturbances on the Earth's surface are associated with MHD disturbances in these flows and penetrate into the magnetosphere from the Solar wind.

The first part of the present study is devoted to the determination of MHD disturbances spectral features caused by the type of plasma flow. When taking into account the possibility of penetration of these disturbances into the magnetosphere, ground-based lowfrequency disturbances should contain information about the type of plasma solar flow approaching the magnetosphere. This is necessary for performing the second part of the study in which the type of disturbed solar wind flow coming up to the magnetosphere. It was established on the base of wavelet spectra components analysis of the geomagnetic field along the high-latitude section of the meridional station chain and parameters of the near-earth space environment. The special attention at the same time is paid to the high-latitude geomagnetic region, where the configuration of the geomagnetic field lines allows direct penetration of LF oscillations from the solar wind into the earth's magnetosphere.

\section{Spectral features of plasma flows MHD disturbances}

It was repeatedly noted earlier that the spectral composition of low-frequency disturbances associated with solar plasma flows is due to their type [3]. In general, the low frequency disturbance is caused by the turbulent regions accompanying the flows. In [4], attention was drawn to the possibility of wide turbulent regions generation by the interaction regions of slow and fast solar flows (CIR - Corotating Interaction Regions). The most geoeffective plasma flows of the magnetic clouds type also often contain a turbulized sheath at the front edge of the cloud [5] that follows the shock wave. It should be noted that most of the research in this area is focused on the study of the turbulence flows nature. At the same time, conclusions about the flow type are often 
not made, and algorithms for flows classifying according to their spectral features are not proposed. The possibility of flow types differentiating by spectral features is demonstrated in the first part of the study.

The study was carried out on materials of 27 cases for magnetic clouds and 15 cases of CIR, obtained from 1998 to 2006 from the Coordinated Data Analysis Web (http://cdaweb.gsfc.nasa.gov). For each event, minute data of solar wind parameters were analyzed: $\mathrm{N}$ (density), $\mathrm{V}$ (speed), and the interplanetary magnetic field (IMF) components $\mathrm{Bx}, \mathrm{By}, \mathrm{Bz}$ in the solar-ecliptic coordinate system, recorded on the ACE spacecraft. Dynamic spectra are obtained as a result of data processing using Fourier transform; dynamic spectra features for different types of solar flows are presented in Table 1.

Table 1. Spectral characteristics of low-frequency disturbances for flows of different types.

\begin{tabular}{|c|c|c|c|}
\hline $\begin{array}{c}\text { Flow } \\
\text { parameter }\end{array}$ & $\begin{array}{c}\text { CIR, } \mathbf{f}\left(\mathbf{E}_{\text {max }}\right) ; \\
\boldsymbol{\Delta} \boldsymbol{\gamma}\end{array}$ & $\begin{array}{c}\text { Magnetic cloud, } \\
\mathbf{f}\left(\mathbf{E}_{\text {max }}\right) ; \boldsymbol{\Delta} \boldsymbol{\gamma}\end{array}$ & $\begin{array}{c}\text { Magnetic cloud } \\
\text { with sheath, } \\
\mathbf{f}\left(\mathbf{E}_{\mathbf{m a x}}\right) ; \boldsymbol{\gamma} \boldsymbol{\gamma}\end{array}$ \\
\hline $\mathbf{B x}, \mathbf{n T}$ & $2-6 \mathrm{mHz} ; 0,2$ & up to $2 \mathrm{mHz} ; 0,1$ & up to $2 \mathrm{mHz} ; 0,4$ \\
\hline $\mathbf{B y}, \mathbf{n T}$ & $2-6 \mathrm{mHz} ; 0,2$ & up to $2 \mathrm{mHz} ; 0,1$ & up to $2 \mathrm{mHz} ; 0,5$ \\
\hline $\mathbf{B z}, \mathbf{n T}$ & $2-6 \mathrm{mHz} ; 0,2$ & up to $3 \mathrm{mHz} ; 0,1$ & up to $3 \mathrm{mHz} ; 0,5$ \\
\hline $\mathbf{N}, \mathbf{c m}-\mathbf{3}$ & $2-6 \mathrm{mHz} ; 0,3$ & up to $3 \mathrm{mHz} ; 0,2$ & $2-8 \mathrm{mHz} ; 0,4$ \\
\hline $\mathbf{V}, \mathbf{k m} / \mathbf{s}$ & $2-8 \mathrm{mHz} ; 0,3$ & up to $2 \mathrm{mHz} ; 0,2$ & $2-8 \mathrm{mHz} ; 0,4$ \\
\hline
\end{tabular}

The main parameters for comparison of obtained spectra were the maximum energy contribution at specific frequencies $\mathrm{f}\left(\mathrm{E}_{\max }\right)$ and the average value $\Delta \gamma$ characterizing by dynamics of the power law describing spectrum in the low frequency (with a period above 23 min) and high frequency part (with a period below 23 $\mathrm{min}$ ). The results analysis indicates the possibility of the solar flow type establishing from its spectral characteristics. Thus, a distinctive feature of CIR-type flows is a registration of intense oscillations in a wide spectral range from 2 to $6 \mathrm{mHz}$ in all parameters.

The spectral composition of magnetic cloud parameters by intense low-frequency oscillations reflects their large-scale structure. The key parameters of these plasma formations are presented in spectral patterns with characteristic frequencies below $3 \mathrm{mHz}$. The magnetic cloud with sheath is also characterized by slow changes in IMF components, but the turbulent nature of cloud sheath provides intensive fluctuations in density and speed throughout the analyzed spectral range up to 8 $\mathrm{mHz}$. In addition, the magnetic cloud with sheath corresponds to the maximum $\Delta \gamma$ power law spectrum, which confirms the presence of a complex internal structure of such plasma formations.

The solution of identifying problem for disturbed solar wind flow type approaching the magnetosphere is not sufficient using the Fourier-based analysis of the geomagnetic field components. This is due to the difficulties of quantifying the consistency of the spectra obtained. Spectral patterns should contain only key features for a successful consistency assessment. This can be achieved by calculating skeletons of studied spectral patterns and their further comparison.

\section{Method for consistency assessment of low-frequency disturbances wavelet spectra using skeleton techniques}

The second part of the study is devoted to the analysis of low-frequency disturbances in the solar wind and on the earth's surface at intervals recorded during the periods from 2000 to 2003 . These are 12 daily magnetospheric storms of varying intensity according to the Dst index (http://spidr.ngdc.noaa.gov/spidr/). This selection included weak and moderate storms (Dst> $-100 \mathrm{nT}$ ), strong storms (Dst> -200 nT) and extreme storms (Dst <$200 \mathrm{nT}$ ). Each interval contained 3-hour data fragments corresponding to geomagnetic storm phases: pre-storm state, initial and main phase. For the disturbances analysis, minute data component of the geomagnetic field from seven observatories of the IMAGE network (http://www.geo.fmi.fi/image) within 106-109 geomagnetic meridian (Table 2) and data of solar wind parameters were used: IMF Bz vertical component, flow density $\mathrm{N}$, pressure $\mathrm{P}$ and electric field $\mathrm{VBz}$ (http://cdaweb.gsfc.nasa.gov).

Table 2. List of selected stations.

\begin{tabular}{|c|c|c|c|c|c|c|c|}
\hline \multirow{2}{*}{$\begin{array}{l}\text { Station } \\
\text { number }\end{array}$} & \multirow{2}{*}{$\begin{array}{c}\text { Abbrev } \\
\text { iation }\end{array}$} & \multirow{2}{*}{ Full name } & \multicolumn{2}{|c|}{$\begin{array}{c}\text { Geographical } \\
\text { coordinates }\end{array}$} & \multicolumn{2}{|c|}{$\begin{array}{l}\text { Geomagnetic } \\
\text { coordinates }\end{array}$} & \multirow{2}{*}{$\begin{array}{c}\text { L-sheath, } \\
\text { Re }\end{array}$} \\
\hline & & & \begin{tabular}{|l|} 
Latit \\
ude
\end{tabular} & $\begin{array}{l}\text { Longitu } \\
\text { de }\end{array}$ & $\begin{array}{l}\text { Latit } \\
\text { ude l }\end{array}$ & \begin{tabular}{|l|} 
Longitu \\
de
\end{tabular} & \\
\hline 1 & HOR & Hornsund & 77.00 & 15.60 & 74.13 & 109.59 & 13.42 \\
\hline 2 & BJN & Bear Island & 74.50 & 19.20 & 71.45 & 108.07 & 9.58 \\
\hline 3 & KEV & Kevo & 69.76 & 27.01 & 66.32 & 109.24 & 6.68 \\
\hline 4 & MAS & Masi & 69.46 & 23.70 & 66.18 & 106.42 & 6.11 \\
\hline 5 & SOD & Sodankylä & 67.37 & 26.63 & 63.92 & 107.26 & 5.14 \\
\hline 6 & OUJ & Oulujärvi & 64.52 & 27.23 & 60.99 & 106.14 & 4.22 \\
\hline 7 & UPS & Uppsala & 59.90 & 17.35 & 56.51 & 95.84 & 2.78 \\
\hline
\end{tabular}

Data processing was performed using wavelet transform and subsequent post-processing of wavelet patterns results. The fourth order Daubechies function was chosen as a base wavelet in numerical experiments [6-11, 14-18]. Scale coefficients of wavelet transform were considered in the range from 4 to 15 , which corresponds to the range of the studied oscillation periods from $120 \mathrm{~s}$ to $450 \mathrm{~s}$. The excess spectral information presented in the case of using the wavelet transform technique is reduced by plotting only the positions of the spectrum maxima on the chart $[8,12]$. In this case, the structure of the "wavelet-skeleton" is formed, which allows to focus on the key features of spectra dynamics [7-8, 13].

The first type skeleton with a vertical orientation "|" is the fastest process of changing the oscillations mode. At high-latitude stations under the auroral oval this may indicate a movement (registration) of the boundary between the polar cap region and the auroral oval. At subauroral stations - registration of the border between the auroral oval and subauroral area.

The skeleton of the second type with an inclination to the right "/" is the oscillation energy dissipation process with the transition from range of high-frequency pulsations to range of low-frequency ones. This may indicate a decrease in the level of energy introduced into the oscillatory system from an external source. 
The third type skeleton with an inclination to the left $" \mid "$ is the process of pumping oscillation energy with a transition from range of low-frequency pulsations to range of high-frequency ones. It may indicate an increase in the level of energy introduced into the oscillatory system from an external source. The skeleton absence in a given time interval can be interpreted as the registration of an oscillations stable mode that is not distinguished by amplitude and frequency in the scale (frequency) range under study. The considered dynamic of disturbance spectra components of the geomagnetic field and solar wind parameters in the context of this study can be interpreted as a classification feature for identifying plasma flows. In addition, based on physical considerations, it should be expected that the components dynamic for disturbance spectra of the solar wind plasma flows parameters manifest themselves in the MHD spectra of high-latitude stations magnetograms. This hypothesis is tested in the work.

Note that step between neighboring skeletons (excluding extreme ones, the appearance of which is caused by the edge effect) indicates the characteristic time scale for the frequency range under study. It is on average $\sim 25-30 \mathrm{~min}$ for wavelet-scale coefficients from 4 to $15(2-8 \mathrm{mHz})$. So the restructuring of the entire oscillatory system occurs at such an interval in a given frequency range. A sample of skeletons in latitude for calculating $D x n$ is formed within a half-hour time window. Reliably matching skeletons are those for which the value of $D x n$ exceeded $70 \%$. Figure 1 shows an example of Dxn calculating with an indication of the calculated time delays $\Delta \mathrm{t}$, which allow identifying the type of geo-efficient solar plasma flow (in this case, the "magnetic cloud"), which is associated with the geomagnetic storm occurrence.

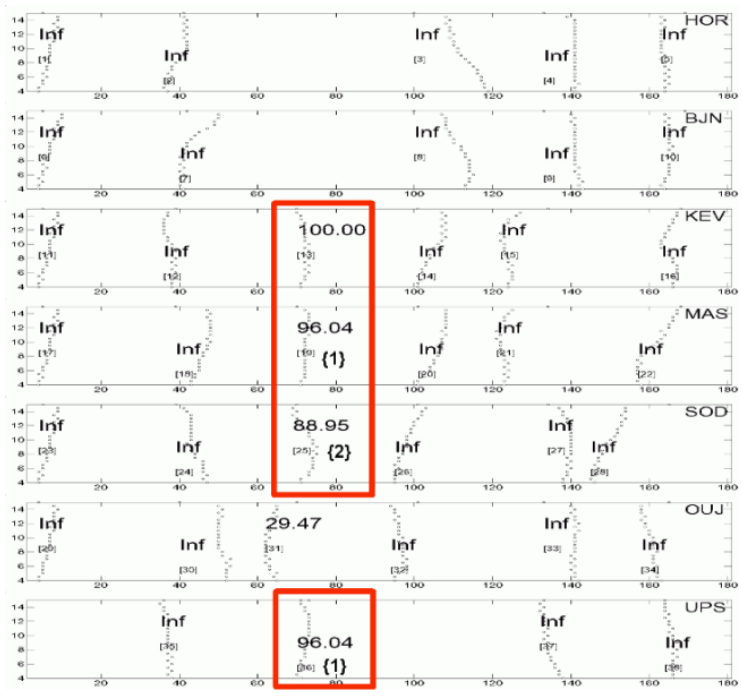

Fig. 1. The $D x n$ value calculating example for skeletons pairs of the geomagnetic field horizontal component at all analyzed stations during the three-hour interval within storm main phase caused by a magnetic cloud $05 / 23 / 2000$. The delay time $\Delta t$ is indicated in curly brackets. Skeletons that were not included in the sample are marked with the "Inf" symbol, the analyzed skeletons are marked with a red marker. The abscissa axis shows time in minutes; the ordinate axis shows scale wavelet coefficients.

\section{Results of numerical experiments and discussion}

The above skeleton technique was applied to the analysis of the interplanetary space main parameters in order to estimate the magnetosphere reaction time in the geomagnetic field horizontal component oscillations at different latitudes on the disturbance approaching it. Vertical component $\mathrm{Bz} \mathrm{IMF}$, concentration $\mathrm{N}$, dynamic pressure $\mathrm{NV}^{2}$ and electric field $\mathrm{VBz}$ of the solar wind flow were chosen as the most geoeffective agents promoting and subsequently accompanying the development of the magnetospheric storm. The time shift definition (in minutes) between the pairs of the "the current station skeleton - the flow parameter skeleton" was established by searching of minimum standard deviation. The shifts which reflect the magnetosphere reaction times are presented in Table 3 for each phase under study ( $\mathrm{p} 1$ is pre-storm state, $\mathrm{p} 2$ is initial phase, p3 is main phase) of analyzed storms, consistently for all stations (from 1 - most northern, to 7 - southernmost). The cases where station skeletons could not unambiguously compare with flow parameters skeletons are marked with dashes. The name of some events in the table 3 is indicated with a question mark. Such cases correspond to the situation when the algorithm for quantifying skeletons consistency of the station spectra among themselves and the spectra of the solar wind parameters provided the Dxn parameter in the range of $50-70 \%$. Since it was previously stated that only those for which the $D x n$ value exceeded $70 \%$ are considered to be reliably consistent skeletons, the events with a "?" sign should be considered less precisely defined.

The disturbance manifestations analysis (see Table 3) for interplanetary space parameters in disturbed horizontal geomagnetic field showed the following features of magnetosphere reaction:

1) Three hours before the storm development ( $\mathrm{p} 1$ is pre-storm state) the magnetosphere reaction time at high latitudes ( 5 minutes on average) is predominantly shorter than the reaction time in the sub-auroral zone (11 minutes on average). Such a result can be interpreted from the standpoint of the generally accepted theory of the particles penetration into the magnetosphere. Particles can penetrate into the polar cap region and fragmentarily into the auroral oval region directly from the solar wind, establishing a new oscillation mode of external magnetosphere lines.

2) At the time of storm initial phase (p2), the magnetosphere reaction time from polar to subauroral latitudes differs slightly. Most likely, this is caused by the overall situation of the geomagnetic field lines compression under pressure of the solar flow and the DCF current growth on magnetosphere surface, which contributes to the synchronization of oscillations in the interplanetary medium with magnetosphere.

3) In the storm main phase (p3) under the action of CIR-type flows, the magnetosphere reaction time at high latitudes (average $4 \mathrm{~min}$ ) is predominantly shorter than the reaction time in the sub-auroral zone (average 12 min). Under the action of flows such as a magnetic cloud with sheath and/or shock wave, the magnetosphere 
Table 3. The reaction estimation of the magnetosphere to interplanetary disturbance according to the results of wavelet analysis of solar wind parameters (the description is given in the text).
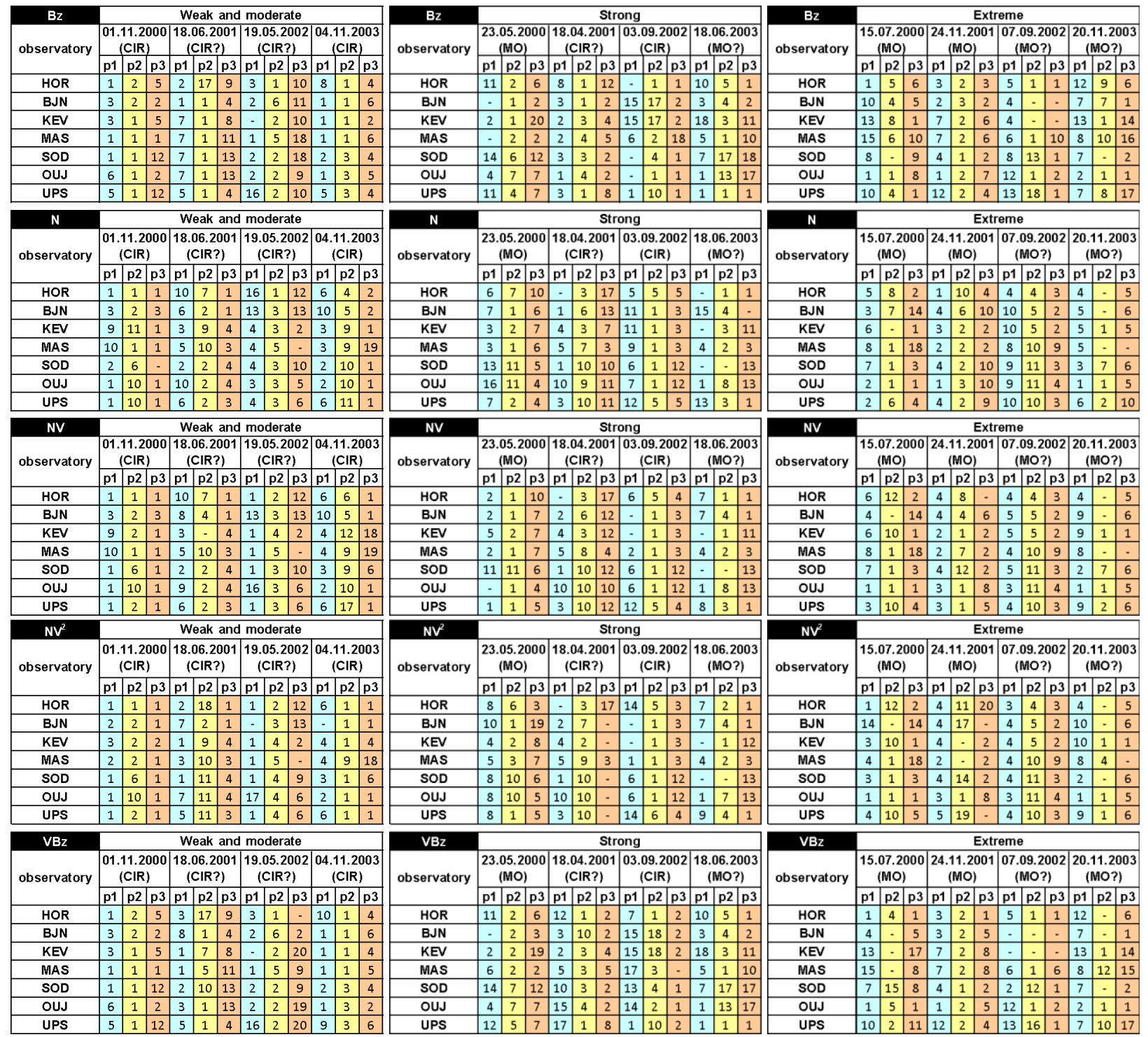

reaction time in the polar and subauroral latitudes differs slightly. It is possible that such a difference is caused by different geometry of the magnetospheric force lines curved under the action of different flows, which leads to different moments of oscillation synchronization at different latitudes.

\section{Conclusion}

The research applied importance is a development an algorithm for calculating the quantitative assessment of the skeletons consistency for stations spectra among themselves and the solar wind parameters spectra. With the help of a computational tool created on the basis of an algorithm, the disturbance spectra reflection of solar wind parameters corresponding to plasma flows in the spectra of observatory magnetograms is demonstrated. It is noted that at the main phase geomagnetic storms contains a spectral trace of the solar wind plasma flow disturbances approaching the magnetosphere. Taking into account that energy spectrum of space oscillations and magnetic field parameters recorded on a spacecraft reflects the internal structure of the corresponding plasma formation, we conclude that it is possible to establish the type of geoeffective solar flow from ground-based observations. It was demonstrated that the dynamics of disturbed spectra components of solar wind parameters corresponding to plasma flows manifest themselves in the MHD spectra of high-latitude stations magnetograms. The method proposed in the paper and computing tool based on it can be used for ground-based diagnostics of solar plasma flows types.

\section{References}

1. A.V. Guglielmi, V.A. Troitskaya, Geomagnetic pulsations and magnetosphere diagnostics (Moscow: Ed. Nauka, 208, 1973) (in Russian) 
2. N.A. Barkhatov, S.E. Revunov, Zh.V. Smirnova, O.T. Cherney, E.A. Semahin, Neural Network Classification of Space Plasma Parameter Discontinuities, International Journal of Advanced Trends in Computer Science and Engineering 9, 3 (May-June 2020)

3. A. Wawrzaszek, W.M. Macek, Observation of the multifractal spectrum in solar wind turbulence by Ulysses at high latitudes, J. Geophys. Res. 115, A07104 (2010), DOI: 10.1029/2009JA0151763

4. N.A. Barkhatov, V.G. Vorobjev, S.E. Revunov, O.M. Barkhatova, E.A. Revunova, O.I. Yagodkina, Neural network classification of substorm geomagnetic activity caused by solar wind magnetic clouds, Journal of Atmospheric and Solar-Terrestrial Physics 205, $105301 \quad$ (2020), DOI: 10.1016/j.jastp.2020.105301

5. K. Steed, C.J. Owen, P. Démoulin, S. Dasso, Investigating the observational signatures of magnetic cloud substructure, J. Geophys. Res. 116, A01106 (2011), DOI: 10.1029/2010JA015940

6. I. Daubechies, Ten Wavelet Lectures (Izhevsk: Ed. NIC «Regular and chaotic dynamics», 464, 2001) (in Russian)

7. N.M. Astafieva, Wavelet analysis: theory and application examples, Advances in the Physical Sciences 166, 11, 1145-1170 (1996)

8. N.A. Barkhatov, S.E. Revunov, Z.V. Smirnova, O.T. Cherney, Observing of polar cap dynamic by Greenland chain of magnetic stations, International Journal of Emerging Trends in Engineering Research 8, 5, 15521555 (May 2020)

9. Yu.V. Manakova, K.A. Pekhteleva, N.A. Barkhatov, S.E. Revunov, Space-time analysis of perturbations of the $\mathrm{Pc} 4-5$ range during periods of magnetic storms by the correlationskeleton method, Vestnikof Minin University, 1, 1-6 (2016)

10. 1 N.A. Barkhatov, Development of methods for predicting the geomagnetic state of the magnetosphere based on the search for fundamental laws of solar-terrestrial relationships, Vestnikof Minin University, 2, 111 (2013)

11. 1 N.A. Barkhatov, S.E. Revunov, A.B. Vinogradov, The classification algorithm for MHD wavelet-skeleton spectral patterns of geoeffective plasma flows in the solar wind, Vestnikof Minin University, 3, 1-6 (2014)

12. 1 S.E. Revunov, S.I. Kuznetsov, O.M. Barkhatova, E.A. Revunova, The problem of connection of observer's mind with quantummechanical description of physical reality, Vestnikof Minin University 7, 3, 14 (2019)
13. 1 N.A. Barkhatov, V.G. Vorobjev, S.E. Revunov, O.M. Barkhatova, O.I. Yagodkina, Substorm Activity and Orientation of the Front of a Shock Wave of an Interplanetary Magnetic Cloud, Geomagnetism and Aeronomy 59, 4, 398-406 (2019)

14. 1 N.A. Barkhatov, E.A. Revunova, O.M. Barkhatova, R.V. Romanov, S.E. Revunov, Studying the relationship of localization parameters of solar sources of magnetic clouds with their characteristics and substorm activity, Solar-Terrestrial Physics 5, 3, 59-67 (2019), DOI: $10.12737 /$ stp-53201907

15. 1 N.A. Barkhatov, S.E. Revunov, M.V. Mukhina, M.L. Gruzdeva, O.T. Cherney, Z.V. Smirnova, Establishing the orientation of shock wave plane of solar wind magnetic cloud for conclusions about the level of auroralsubstorm activity, JP Journal of Heat and Mass Transfer 17, 1, 195-202 (June 2019), DOI: 10.17654/HM017010195

16. 1 N.A. Barkhatov, S.E. Revunov, O.M. Barkhatova, E.A. Revunova, N.V. Kosolapova, Algorithm for Detecting an Extreme Solar Flare of September 6, 2017 from its Geomagnetic Precursors, Radiophysics and Quantum Electronics 62, 10, 651-656 (March 2020) (Russian Original 62, 10, October 2019), DOl 10.1007/s11141-020-10010-6

17. 1 N.A. Barkhatov, D.S. Dolgova, E.A. Revunova, Dependence of the geomagnetic activity on the structure of magnetic clouds, Geomagn. Aeron. 59, 1, 16-26 (2019)

18. 1 O.M. Barkhatova, N.V. Kosolapova, N.A. Barkhatov, S.E. Revunov, Synchronization of geomagnetic and ionospheric disturbances over Kazan station, Solar-Terrestrial Physics 3, 4, 58-66 (2017), DOI: 10.12737/stp-34201706 